\title{
Surveillance Strategies During Low Ebola Transmission in a District in Sierra Leone
}

\author{
Allison M. Connolly*, Alyssa J. Young, Brooke Mancuso, Mary-Anne Hartley, Adam \\ Hoar, Guddu Kaur, John Mark Esplana, Laura Fisher and Anh-Minh A Tran
}

GOAL, Dublin, Ireland

\section{Objective}

Describe the evolution of Ebola Virus Disease (EVD) surveillance from a largely reactive system structured primarily around responding to reports of illness and death, to one that was more methodical, proactive and comprehensive.

\section{Introduction}

Port Loko District has had over 1400 confirmed EVD cases during this outbreak. However, transmission declined rapidly in early 2015; by mid-April, a few weeks had passed with no known cases. Simultaneously, reporting of sick persons had plummeted across the district and the number of deaths reported in some areas was fewer than expected. These circumstances signaled the need for the EVD surveillance system to broaden its focus from using district surveillance officers (DSOs) to respond to reports of ill and deceased persons (hereafter, "sick and death alerts") to a more proactive and comprehensive system that relied strongly on community engagement and surveillance through existing structures such as Peripheral Health Units (PHUs), schools and local authorities. While the involvement of local authorities and the community had been central to reporting suspected EVD cases earlier in the outbreak, the decrease in alerts suggested that engagement was diminishing. The reopening of schools and reemergence of the primary healthcare system provided opportunities to decentralize surveillance and strengthen the involvement of these structures. The primary objective was improving EVD surveillance, but the effort was also used to bolster routine surveillance, in preparation for implementating Integrated Disease Surveillance and Reporting.

\section{Methods}

- GOAL, WHO and district staff developed a Standard Operating Procedure to guide surveillance activities during periods of low EVD transmission.

- DSOs received 5 sessions of training, in addition to mentoring in the field.

- The district has 11 chiefdoms, divided into a total of 162 sections. A qualitative survey was developed for DSOs to assess and validate the engagement of key partners such as local authorities, social mobilizers and contact tracers in surveillance-related activities in each section.

- Tools were used to assess surveillance and provide training at PHUs, schools and border areas.

- Expected mortality rates (pre-EVD outbreak) were compared to the number of death alerts received. Sections with the highest levels of under-reporting were prioritized.

- PHU staff were trained on the importance of using diagnostic tests for malaria, and following up on patients to ensure that no EVD cases were missed due to incorrect diagnosis or co-infection. Registers were used to record symptoms for all ill patients, as well as test results, treatment and follow up for those with malaria. Weekly reports to the district of reportable diseases were checked for completeness. Health screening for arriving clients and basic infection prevention and control (IPC) protocols were verified, as were knowledge of the case definition and reporting expectations for suspected EVD. Community engagement efforts were discussed.

- DSOs were trained to review trends in absenteeism at schools as an indicator of potential disease outbreaks. Also, to ensure active surveillance and IPC were done correctly.

- In villages bordering other districts, enhanced cross-border monitoring was used as an essential tool to minimize risk of EVD infiltrating from other districts.

\section{Results}

- DSOs needed more training than anticipated to implement new practices. On-site mentoring was a critical supplement to formal training.

- After initial difficulties adapting to new expectations for using the registers, documentation improved, which permitted monitoring the implementation of the protocol for malaria patients, as well as active case search for numerous conditions.

- Follow-up actions for symptomatic children were unclear to school staff. Concerns that direct follow up by DSOs might jeopardize school participation indicated that community-supported alternatives were crucial.

- Using attendance registers to look for disease trends was difficult since attendance hadn't yet stabilized due to the outbreak itself.

- Use of expected vs reported deaths to prioritize surveillance efforts is now routine in the district.

\section{Conclusions}

This system has been effective in guiding district surveillance activities. Additional technology, data and staff have allowed its implementation to become more targeted. Capacity for routine surveillance in the post-Ebola period has improved.

\section{Keywords}

surveillance; Ebola; EVD; Sierra Leone; Community; Malaria

\section{Acknowledgments}

GOAL acknowledges the support of WHO, CDC, Port Loko District Health Managment Team and the District Ebola Response Team.

\author{
*Allison M. Connolly \\ E-mail: allison.connolly@thepalladiumgroup.com
}

